\title{
Appréhender et s'adapter
}

Les goémoniers bretons face aux changements climatiques

Understanding and adapting: seaweeds harvesters in Brittany facing climate change

\section{Clément Garineaud}

\section{OpenEdition}

\section{Journals}

\section{Édition électronique}

URL : https://journals.openedition.org/ethnoecologie/6864

DOI : 10.4000/ethnoecologie.6864

ISSN : 2267-2419

\section{Éditeur}

Laboratoire Éco-anthropologie

\section{Référence électronique}

Clément Garineaud, «Appréhender et s'adapter », Revue d'ethnoécologie [En ligne], 19| 2021, mis en ligne le 30 juin 2021, consulté le 17 décembre 2021. URL : http://journals.openedition.org/ ethnoecologie/6864; DOI : https://doi.org/10.4000/ethnoecologie.6864

Ce document a été généré automatiquement le 17 décembre 2021.

\section{(i) $\odot$

Revue d'ethnoécologie est mis à disposition selon les termes de la licence Creative Commons Attribution - Pas d'Utilisation Commerciale - Pas de Modification 4.0 International. 


\section{Appréhender et s'adapter}

Les goémoniers bretons face aux changements climatiques

Understanding and adapting: seaweeds harvesters in Brittany facing climate change

Clément Garineaud

\section{L'étude des savoirs locaux prémisse à une anthropologie du changement climatique}

1 Les ethnologues abordent la question du changement climatique et sont en mesure de prendre part aux réflexions globales notamment sur les formes d'adaptation des communautés (Baer \& Reuter 2015). La problématique de l'adaptation locale au changement global reste peu traitée, dans la mesure où elle oblige à une connaissance approfondie des groupes étudiés et de leurs visions du monde. Pourtant, c'est une réalité émergente sur le terrain pour les ethnologues (Crate \& Nuttall 2009: 9). À partir de matériaux ethnographiques et ethnoécologiques traitant des rapports entre l'environnement et une petite communauté de collecteurs d'algues en Bretagne, cet article interroge la manière dont se formulent localement les savoirs météorologiques et la perception des changements locaux et globaux par l'étude des savoirs locaux et des mécanismes d'adaptation.

2 Le discours scientifique et politique global sur le changement climatique dans lequel est produite la connaissance scientifique se focalise majoritairement sur la question de l'adaptation de façon prescriptive (Crate \& Nuttall 2009: 9). Bien que la notion d'adaptation ${ }^{1}$ puisse être ambiguë comme le démontre Godard dans le cas des changements climatiques (Godard 2010), «par cette interdisciplinarité [...] elle devient un outil pour penser le rapport entre l'environnement et la société dans une perspective systémique d'actions réciproques» (Simonet 2009: 400). Aujourd'hui, l'appropriation immatérielle et matérielle de la nature est influencée par des facteurs socio-économiques locaux complexes. Elle est également soumise à des dynamiques globales contradictoires entre politiques économiques et politiques de la conservation 
de la nature (Dahou 2018). Pour cette raison, l'apport de la political ecology est indéniable pour saisir les dimensions institutionnelles et les initiatives politiques liées aux changements climatiques. L'anthropologie des changements climatiques se donne comme objectif d'explorer la construction de ces changements dans ses dimensions culturelles, politiques et sociales (Dove 2014). Toutefois, la question de l'adaptation ne doit pas se limiter aux systèmes socio-écologiques à grande échelle (Adger et al. 2005, Ojea et al. 2017), mais bien intégrer la dimension locale des changements climatiques. $\mathrm{Au}$ travers des perceptions, des connaissances et des expériences individuelles et collectives, l'anthropologie des changements climatiques permet de comprendre comment est compris le changement global à l'échelle locale (Crate \& Nuttall 2009, Dove 2014). Elle va également interroger le rapport changement local versus changement global et décrire l'évolution parallèle de ce rapport et les réponses sociales misent en place. Dove traduit ces approches sous trois constats: les changements climatiques ont une réalité locale malgré leur caractère global; les climats sont des agents à part entière qui co-évoluent avec les sociétés humaines; et les savoirs et les perceptions du climat forment un phénomène social qui affecte la façon dont les sociétés humaines s'adaptent ou atténuent les changements climatiques (Dove 2014). Pour lui, l'analyse des systèmes de savoirs apparaît comme un moyen de saisir la réalité locale du changement climatique, puis d'examiner la façon dont des populations locales s'y adaptent (Ibid).

3 Les changements globaux se matérialisent localement par des modifications des écosystèmes affectant potentiellement les pratiques des acteurs. Le premier constat de Dove trouve un écho du point de vue écologique sur le littoral breton comme d'autres écosystèmes côtiers qui, affectés par les changements globaux voient leurs capacités à maintenir la biodiversité présente et à produire des services pour les populations se réduire (GIEC 2014, Pecl et al. 2017). Alors que le littoral breton abrite une diversité d'espèces marines, dont des forêts d'algues parmi les plus diversifiées au monde en termes d'espèces (Keith et al. 2014), la température de l'eau de surface a augmenté en moyenne de $0,35^{\circ} \mathrm{C}$ par décennie au cours des vingt dernières années, soit nettement plus que ce qui est observé globalement ${ }^{2}$ (Gallon et al. 2014). Des études phycologiques à l'échelle de la Bretagne, européennes et mondiales montrent l'influence du changement climatique sur les communautés d'algues provoquant le déplacement ou la disparition de certaines espèces pourtant au centre des activités de récolte locales (Gallon et al. 2014, Pecl et al. 2017, Raybaud et al. 2013). En Bretagne, l'accroissement des températures pourrait limiter la reproduction des ceintures de Laminaria digitata (Figure 4), espèce centrale dans l'activité goémonière entraînant une diminution de la biomasse et un remplacement des espèces (Gallon et al. 2014, Valero et al. 2011). Les scénarios climatiques proposés par le GIEC prévoient, en outre, une augmentation de la fréquence et de l'intensité des tempêtes, avec des conséquences fortes sur la biodiversité côtière (GIEC 2014). Ce changement dans les régimes de tempête pourrait conduire à une réduction de la quantité et de la qualité des algues en Bretagne. Les transformations qui affectent les champs d'algues, support depuis plusieurs siècles d'une activité économique ayant façonnée ce littoral et la vie de ses habitants pourraient mettre à mal une activité patrimoniale et aujourd'hui économiquement importante (Collectif 2017). Actuellement, les goémoniers récoltent en bateau plusieurs espèces d'algues, pour la production d'épaississant-gélifiants à destination de l'industrie agroalimentaire ou cosmétique. Ils vivent et travaillent en étroite relation avec les milieux côtiers. 
Si le milieu maritime se révèle être un espace longtemps négligé par l'ethnobiologie (Narchi et al. 2014), l'anthropologie maritime a investi cet espace avec de nombreuses approches (Artaud 2018). Notre étude s'inscrit dans quelques-unes comme la diversité des cultures ou des expériences de la vie en mer (Geistdoerfer 2004, Jorion 1983), le système pêche (Acheson 1981, Geistdoerfer 1974) et la richesse des savoirs des " gens de mer " (Johannes 1981). Dans ce domaine, l'anthropologie maritime a fait la démonstration depuis longtemps dans différents contextes géographiques et sociaux, que les communautés de pêches disposent de savoirs locaux et techniques adaptés à leur environnement et évolutifs pour faire face aux changements. (Dond \& Manyungwa-Pasani 2018, de Freitas 2018, Folke et al. 2002, etc.). Aujourd'hui, la mer est investie par d'autres groupes sociaux et notamment les scientifiques sur les questions de conservation des ressources marines, sur les moyens et les politiques de gestion et les changements climatiques. Ce n'est pas sans influence, débats et controverses sur les pratiques et les expériences des pêcheurs (Artaud 2011, Doyon \& Sabinot 2015).

5 Les collecteurs d'algues en bateau ne sont-ils pas en effet des marins parmi d'autres interagissant avec le milieu naturel et des communautés scientifiques? (Arzel 1987, Garineaud 2017, Jacquin 1980) Comme de nombreux groupes autochtones ou de professionnels ayant un lien privilégié avec l'océan, les goémoniers bretons possèdent un cortège de savoirs locaux ${ }^{3}$ (Roué 2002), - sur les courants, le fond marin et les algues - indissociables des pratiques et contribuant à leur appropriation de l'espace maritime tant sur plan sensoriel que spatial (Garineaud 2015, Garineaud et al. 2016). Leurs activités se pratiquent dans des milieux très dynamiques avec forte variabilité de la ressource (Acheson 1981), ils sont donc des observateurs privilégiés de l'environnement et de ces changements. Le littoral breton, comme nous l'avons explicité précédemment est soumis aussi bien aux enjeux de conservation avec la présence du Parc naturel marin d'Iroise et qu'aux effets des changements globaux. Ces deux champs intéressent une communauté scientifique implantée localement interagissant avec les goémoniers. Tous ces éléments, nous invitent à décrire et à comprendre : comment les goémoniers appréhendent-ils cette variabilité dans leurs pratiques et dans leurs savoirs? Quel rapport ont-ils entre cette variabilité et ce qu'ils perçoivent de la réalité locale des changements climatiques? Comment se traduisent l'adaptation et la réalité des changements climatiques? Comment l'adaptation des savoirs et des savoir-faire s'inscrit dans des mécanismes plus larges d'interactions avec les acteurs scientifiques et industriels?

6 La réflexion ${ }^{4}$ présentée s'appuie sur des données collectées entre 2012 et $2017^{5}$ sur les côtes de la Bretagne, principalement dans le Finistère nord, entre Brest et Roscoff où se concentre la majorité des activités de récolte (Figure 1). 
Figure 1 : Localisation du terrain de recherche

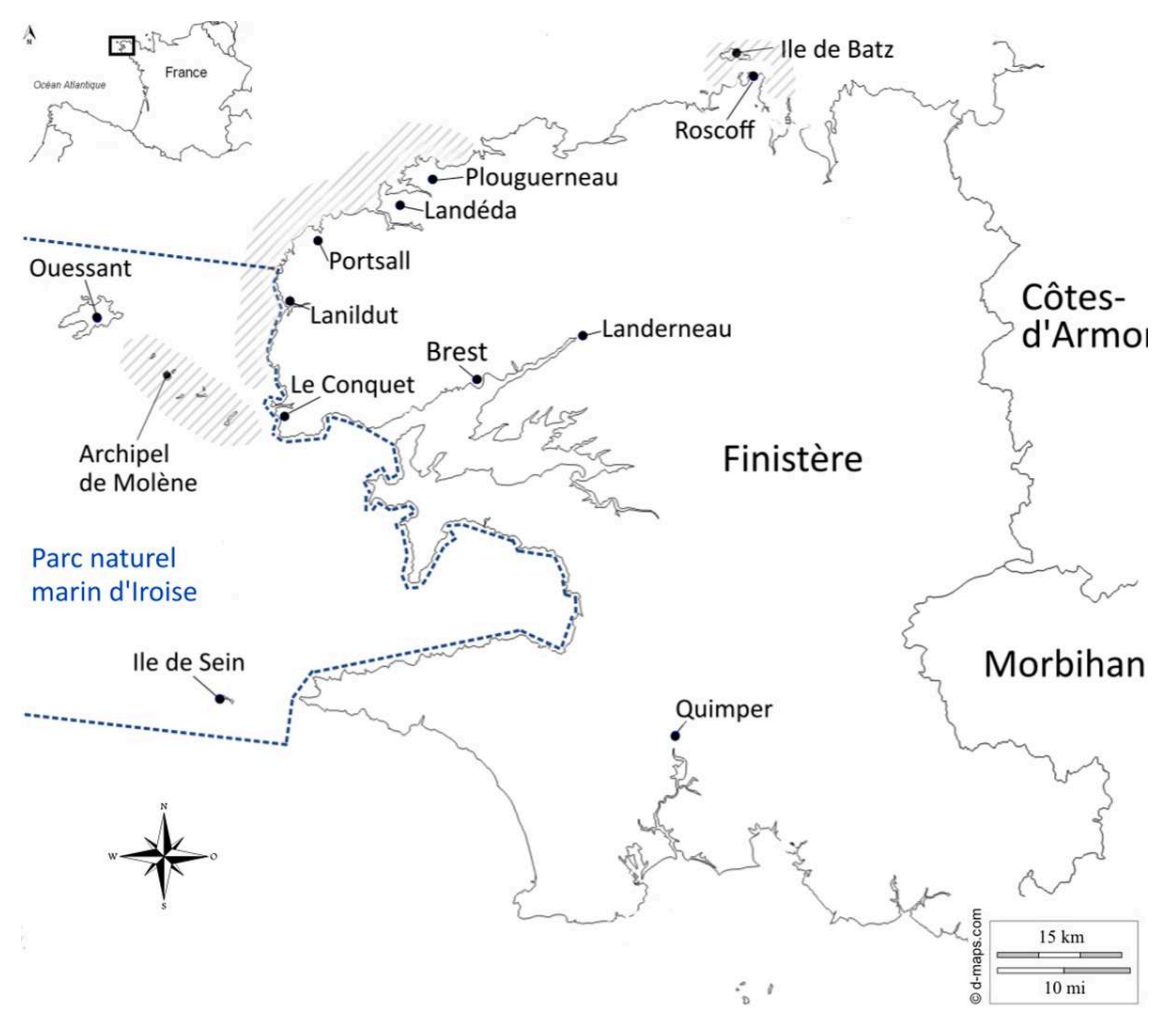

Localisation du terrain de recherche en Bretagne. Les zones hachurées représentent les principaux secteurs de récolte des algues

(c) C. Garineaud

7 Les données ont été recueillies pendant une enquête ethnographique d'environ an et demie sur le terrain. Les informations sont le croisement des observations participantes pendant plusieurs sessions de récolte en mer, des échanges informels par exemple dans les ports lors du déchargement des algues et d'une cinquantaine d'entretiens enregistrés réalisés avec les goémoniers, des biologistes et écologues spécialisés dans l'étude des algues ainsi que des gestionnaires des ressources de l'IFREMER $^{6}$ et du Comité régional des pêches maritimes et des élevages marins ${ }^{7}$. L'enquête a permis de recueillir les techniques de récolte, les savoirs locaux et les visions du monde des collecteurs d'algues. 
Figure 2 : Présentation du corpus de données issu de l'enquête

\begin{tabular}{|c|c|c|c|}
\hline \multicolumn{4}{|c|}{ Présentation du corpus de données } \\
\hline \multicolumn{4}{|c|}{ Détails des entretiens } \\
\hline \multicolumn{4}{|c|}{ Nombre d'entretiens par catégories d'acteurs } \\
\hline \multicolumn{3}{|c|}{ Goémoniers embarqués } & 21 \\
\hline \multicolumn{3}{|c|}{ Anciens Goémoniers } & 6 \\
\hline \multicolumn{3}{|l|}{ Chercheurs } & 7 \\
\hline \multicolumn{3}{|c|}{ Gestionnaires d'espaces et de ressources } & 4 \\
\hline \multicolumn{3}{|l|}{ Industriels } & 3 \\
\hline \multicolumn{3}{|c|}{ Membres d'instances administratives } & 4 \\
\hline \multicolumn{3}{|c|}{ Chargés de mission dans le patrimoine sur les algues } & 3 \\
\hline \multicolumn{3}{|c|}{ Pêcheurs professionnels } & 2 \\
\hline \multicolumn{3}{|l|}{ Agriculteur } & 1 \\
\hline \multicolumn{4}{|c|}{ Détails des observations participantes } \\
\hline Activité & Nombre de sorties & Espèces récoltées lors des sorties & Nombre d'observations \\
\hline \multirow{4}{*}{$\begin{array}{c}\text { Récolte } \\
\text { à partir des bateaux }\end{array}$} & \multirow{4}{*}{5} & Laminaria digitata & 2 \\
\hline & & Laminaria hyperborea & 1 \\
\hline & & Praire en rade de Brest & 1 \\
\hline & & Araignées de mer et poissons en mer d'Iroise & 1 \\
\hline
\end{tabular}

Le nombre d'observations participantes est réduit en raison des contraintes imposées aux professionnels embarqués. Un seul bateau possède une autorisation

(C) C. Garineaud

Notre article s'attache dans un premier temps à décrire l'activité goémonière par l'utilisation deux dimensions fondamentales de métier et de saison. Dans un second temps, nous mettrons en évidence les savoirs des goémoniers qui leur permettent d'appréhender la variabilité du milieu naturel. Leurs interrogations sur la projection à long terme seront l'occasion de saisir la réalité locale des changements climatiques. La saison hivernale 2013-2014, exceptionnelle par les tempêtes qui l'ont émaillée, nous fournit une esquisse de la matérialisation des changements à l'échelle locale et de l'adaptation des goémoniers. L'analyse des réponses nous permet enfin de nous interroger sur la capacité d'adaptation des goémoniers face à des changements plus globaux.

\section{Métiers et saisons : spatialité et temporalité de l'activité goémonière}

9 Aujourd'hui, trente-six navires, soit une quarantaine de goémoniers, sont en activité en France, principalement dans le Finistère Nord, autour de l'archipel de Molène-Ouessant et sur la côte du Conquet à Roscoff (Figure 1).

La pêche artisanale se caractérise par une unité productive "fondée sur le ménage " (Delbos 2006 : 531), contrairement à la pêche industrielle qui est construite sur la firme ou la compagnie, le patron du bateau est embarqué à son bord. Comme l'agriculture, la pêche artisanale véhicule l'image de l'un des derniers métiers que l'on fait par tradition familiale (Ibid). La tradition et la transmission familiale perdurent chez les goémoniers puisque la quasi-totalité d'entre eux a un membre de sa famille travaillant dans la récolte des algues. Sur une quarantaine de professionnels, seuls, deux n'ont aucun 
parent dans la profession et sont devenus goémoniers par opportunité professionnelle après un changement dans leur vie active (retraité de l'armée ou licenciement).

11 Les goémoniers sont un groupe composé d'hommes, âgés de 30 à 70 ans, avec une homogénéité relative dans leurs parcours personnels et professionnels. Tous originaires du Finistère, leur première étape professionnelle est une expérience courte aux côtés du père ou de l'oncle paternel ou maternel. La seconde étape est celle de "l'école de pêche ${ }^{8}$ ", où les jeunes apprennent le matelotage et les bases de navigation et de la pêche (Biget 2005). Certains complètent leurs apprentissages par des formations techniques en mécanique ou en électronique par intérêt personnel et professionnel. La troisième étape est l'obtention du diplôme pour " patronner » un navire après une période de navigation, sur des bateaux de pêche aux casiers, aux filets ou directement comme matelot sur un navire goémonier. Ces expériences permettent la constitution d'un capital économique afin de pouvoir acheter un bateau. Lors de l'achat du bateau, l'ancien propriétaire accompagne quelques jours le nouveau patron. Quelques goémoniers intègrent directement le métier lorsqu'un membre de leur famille est déjà en activité. Le recrutement de l'équipage se fait dans le cercle familial proche : fils, frère ou gendre. Si l'activité sur les bateaux est un univers masculin, les femmes occupaient une place centrale dans le système algue avant la professionnalisation intervenue dans les années $1970^{\circ}$ en récoltant les algues à pied et en s'occupant de la ferme (Arzel 1987). Aujourd'hui, la flottille goémonière ne comprend ni patronne, ni femme matelot et seulement trois femmes ont pratiqué cette activité au cours des vingt dernières années ${ }^{10}$.

12 La pêche goémonière est structurée par les deux dimensions fondamentales identifiées par Paul Jorion (1979) : la saison et le métier. La saison peut correspondre à plusieurs échelles de temps. Il s'agit de l'époque d'une année où il est possible de pêcher une certaine espèce en particulier en grandes quantités, d'une unité de temps de travail pour le pêcheur et d'une unité de compte dans la comptabilité de l'entreprise qu'est un bateau de pêche. Nous identifions ici deux saisons : la saison hivernale et la saison des algues. Le métier est défini par des conditions de travail particulières, une pratique d'engins de pêche mobilisant un ensemble de savoirs techniques et de connaissances empiriques sur le milieu naturel et sur l'espèce dans un objectif de capture de celle-ci (IFREMER 2018, Jorion 1979).

\section{En saison hivernale : la coquille, un complément d'activité indispensable}

13 Au regard de ces définitions, les goémoniers exercent aujourd'hui entre deux et quatre métiers répartis sur deux grandes saisons (Figure 3). Entre les deux saisons, des périodes de carénage du bateau sont prévues. Leurs activités se répartissent majoritairement de la manière suivante sur une année :

«De début mai à fin août on fait les laminaires [Laminaria digitata]. Après, on fait hyperboréa de septembre à mi-novembre au plus tard et après on reprend à partir de mi-janvier ou fin janvier jusqu'à fin mars début avril. Entre mi-novembre et fin février on fait la coquille en rade de Brest. » (Un goémonier expérimenté) ${ }^{11}$ 
Figure 3 : Calendrier du goémonier avec ses métiers, les saisons et les repères qu'il mobilise pour sa récolte

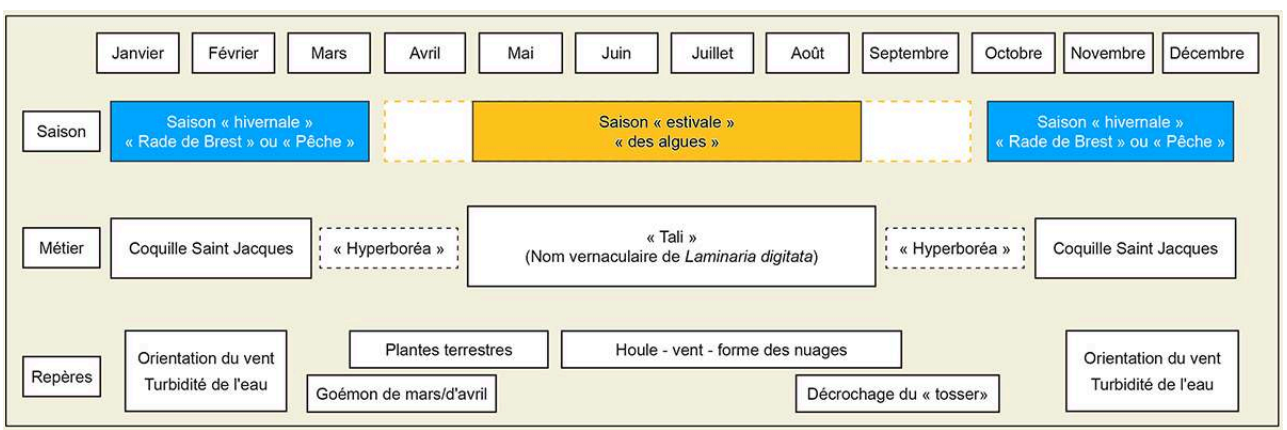

Le calendrier montre les trois métiers et les deux saisons des goémoniers au cours de l'année. Les pointillés indiquent que seule une partie de la flottille est active lors de cette saison ou de ce métier. Les principaux paramètres naturels mobilisés par les goémoniers pour connaître la météo ou prédire l'état de la ressource sont également indiqués

(c) C. Garineaud

14 La saison hivernale s'étend de fin novembre à la mi-mars, période durant laquelle la grande majorité des bateaux exercent le métier de « coquillier » en rade de Brest avec pour cible la coquille Saint-Jacques (Pecten maximus), le pétoncle noir (Mimachlamys varia) et la praire (Venus verrucosa). La réglementation permet à tous les pêcheurs coquilliers de disposer disposent de trente jours de pêche par an qu'ils répartissent selon leurs choix. Si pour les goémoniers le temps de travail annuel est faible, pour la plupart des navires, cette saison représente 40-50\% du chiffre d'affaires annuel d'un navire, elle est donc indispensable. Quelques professionnels se consacrent en fonction de leurs opportunités et de leurs préférences à la pêche de la lotte (Lophius piscatorius) et aux araignées (Maja brachydactyla) aux casiers, ou aux huîtres (Crassostrea gigas).

Tout changement affectant cette activité a donc une influence forte sur l'activité de récolte des algues. Or, des fermetures ou des limitations ont eu lieu depuis 2012 notamment en rade de Brest à cause des efflorescences de microalgue Pseudo-nitzschia, qui produit des toxines amnésiantes rendant les coquillages impropres à la consommation. Ces restrictions ont conduit une diminution des revenus incitant les goémoniers à diversifier leurs activités de récolte des algues. Si ces blooms de phytoplancton résultent d'une multitude de facteurs, leurs récurrences amènent les goémoniers à se questionner sur les modifications. Il est intéressant de remarquer que les dimensions climatiques ne sont pas vues comme fondamentales. La raison majeure évoquée par les professionnels est la pollution de l'eau. Or, les études comme celle de Delegrange et al. (2018) montrent que la disponibilité des éléments nutritifs et les conditions environnementales les températures, vents, précipitations sont intrinsèquement liés.

\section{La saison des algues : le cœur du métier de goémonier}

La saison principale est celle des algues. C'est elle qui distingue les goémoniers des autres pêcheurs puisqu'ils sont les seuls à pratiquer cette activité. Elle est donc constitutive de leur identité professionnelle et plus largement de leur identité sociale (Garineaud 2017). Cette saison est divisée en plusieurs métiers (Figure 3). 
Le métier que tous les goémoniers pratiquent correspond à la récolte de Laminaria digitata (Figure 4) de mai à septembre. La date d'ouverture, les modalités d'exploitation et les règles de gestion sont décidées en commission réunissant tous les acteurs de la filière - goémoniers, industriels, IFREMER, administration (Frangoudes \& Garineaud 2015). Les dates de récolte pour chaque bateau sont établies par les usines de transformation qui achètent les algues dans le cadre d'un contrat confidentiel signé avec le goémonier pour chaque saison. Aujourd'hui, deux entreprises ${ }^{12}$ se partagent le marché. La récolte de Laminaria digitata se fait grâce au « scoubidou » que le goémonier plonge au milieu des algues à récolter (vidéo 1). Grand crochet au bout d'une longue barre de métal, autrefois mis en mouvement à la force des bras, il est mécanisé au début des années 1970 grâce à un bras hydraulique. Par la rotation de l'outil, les algues s'enroulent autour du crochet et de la barre permettant leur arrachage (Garineaud 2015).

Figure 4 : Laminaria digitata (à gauche) et Laminaria hyperborea (à droite)
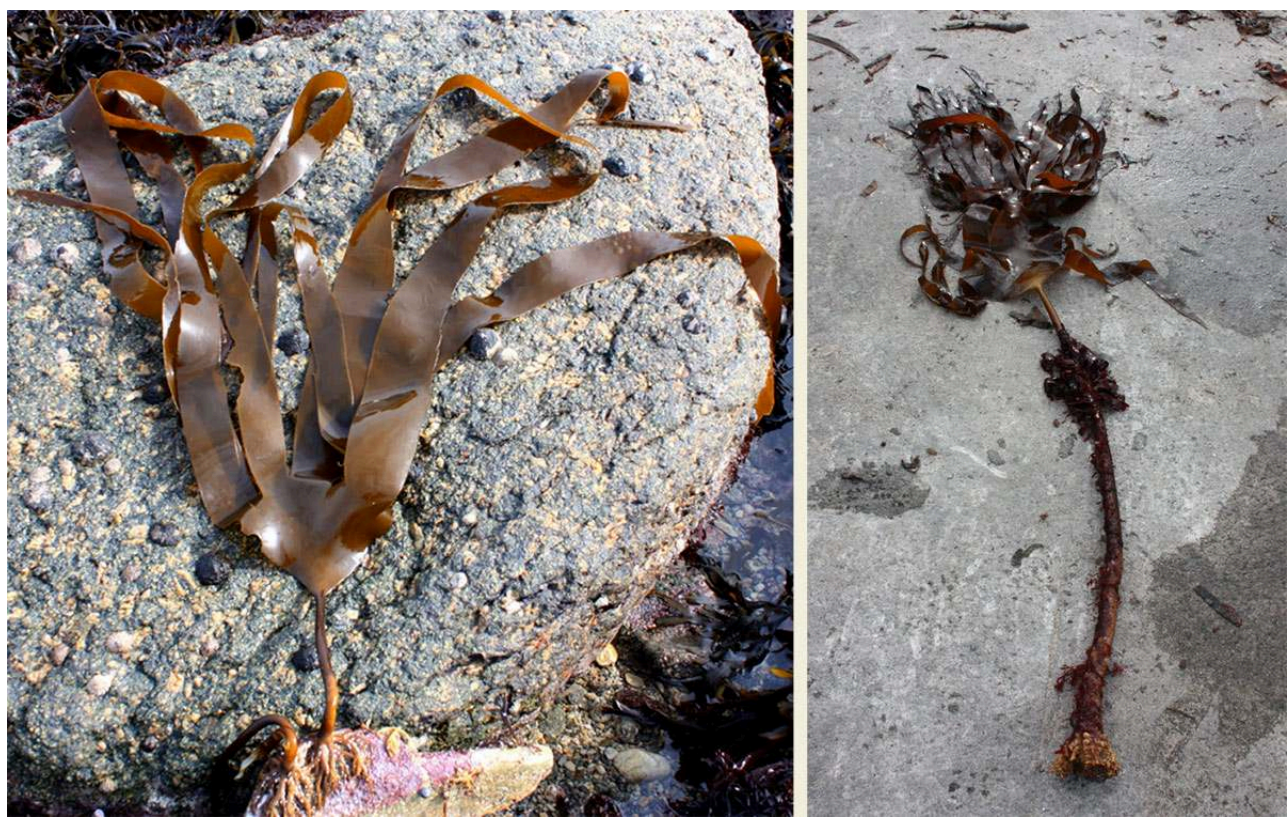

Les deux espèces se distinguent notamment par le stipe court et flexible pour sur L. digitata et long et rigide sur L. hyperborea. II s'agit d'un des paramètres obligeant l'utilisation de techniques différentes (c) C. Garineaud

Le second métier consiste en la récolte de Laminaria hyperborea (Figure 4). Depuis les années 2000, afin de diversifier les revenus et répondre à une demande croissante, sous l'essor de quelques goémoniers, des acteurs industriels (entreprises acheteuses et transformatrice des algues) et de l'IFREMER, la récolte de Laminaria hyperborea se met en place progressivement de février à avril et de septembre à novembre. Avant 2014, moins de dix goémoniers sur la quarantaine récoltent cette algue grâce à une technique inspirée des collecteurs d'algues norvégiens par l'usage du "peigne norvégien" (Figure 6). Mesurant environ un mètre par deux mètres cinquante, il est constitué de cinq «skis » entre lesquels s'intercalent quatre à six doigts de $60-70 \mathrm{~cm}$. Relié par un câble en acier à un bras hydraulique, il est traîné sur le fond à une vitesse d'environ deux nœuds ${ }^{13}$ pendant deux à trois minutes (Garineaud 2015). Si certains professionnels sont incités par les entreprises acheteuses à récolter cette algue, ils refusent pour des 
raisons économiques estimant que ce n'est rentable ou personnelles, car incompatible avec leurs autres activités.

Figure 5 : Un goémonier, le Jean Cani chargé d'algues avec son scoubidou

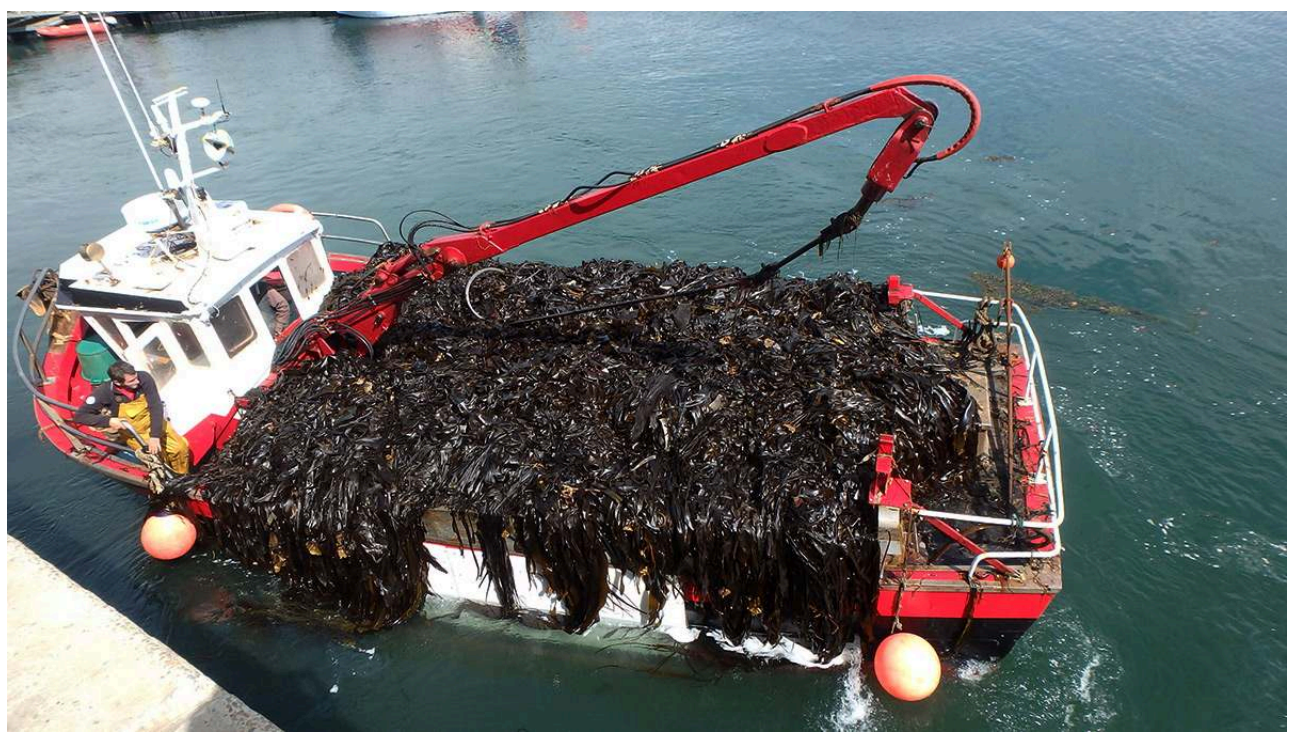

Le bateau chargé s'amarre au quai de déchargement. Sur le tas d'algues est posé le scoubidou, longue tige en acier avec un crochet à la base. Il est attaché au bras hydraulique par un moteur qui permet sa rotation

(C) C. Garineaud

Figure 6 : Le peigne norvégien du goémonier Le Nautilus

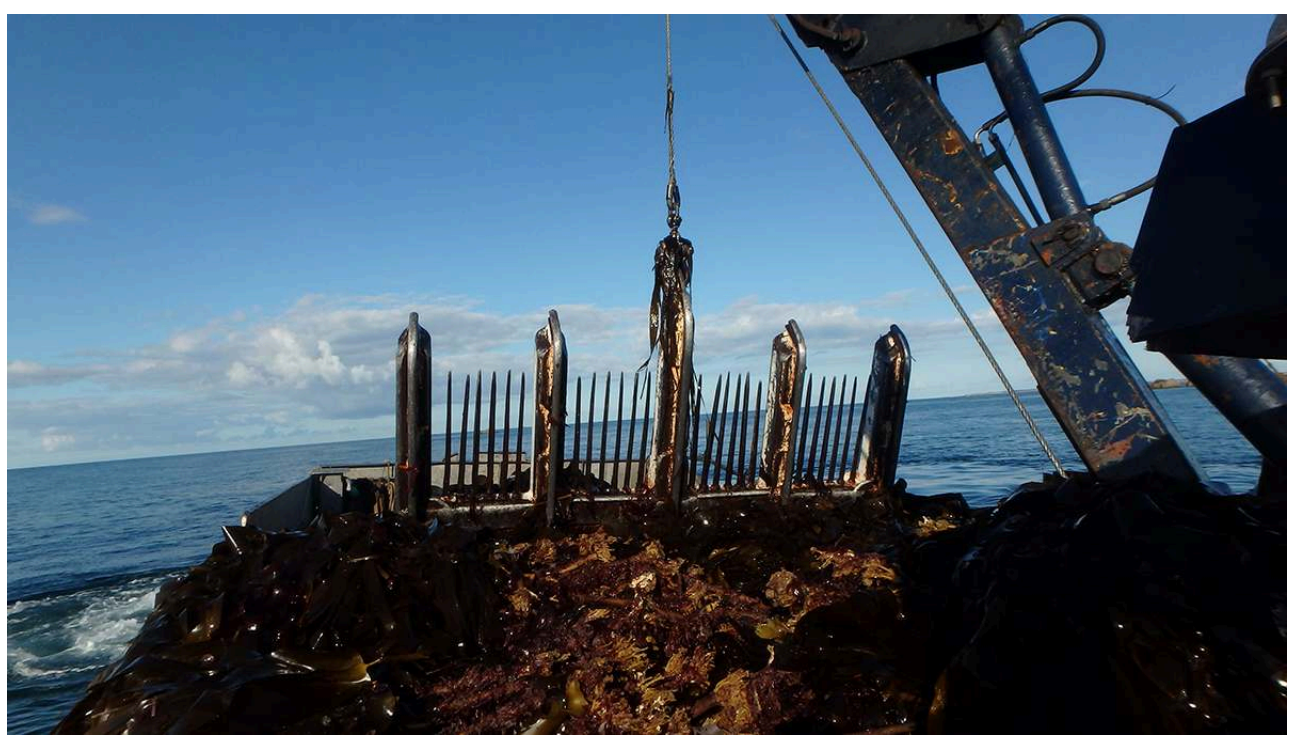

Le peigne norvégien, composé de doigts, est relié par un câble en acier au bras hydraulique (c) C. Garineaud 


\section{De la variabilité de la ressource à la perception de changements locaux}

\section{Des savoirs pour une bonne saison de récolte}

西 variations rapides du temps. Une partie de leurs observations se réfèrent à des activités historiques et des dénominations vernaculaires: "goémon de mars » et " goémon d'avril », désignant les algues qui viennent s'échouer en mars et en avril sur la côte. Jusqu'aux années 1960, très convoitées pour amender les terres et fournir les industries historiques de la soude et de l'iode, elles étaient ramassées et faisaient l'objet de réglementations et de conflits (Arzel 1987, Jacquin 2000). Si ces pratiques ont disparu lors des changements professionnels et sociaux profonds intervenus dans les années 1960-1970 ${ }^{14}$, ces dénominations reflètent la mémoire et le savoir conservés par les goémoniers grâce à une transmission entre générations et trouvent encore aujourd'hui une utilité en tant qu'indicateurs de changements météorologiques. Les plus anciens goémoniers sont ainsi capables de prédire la localisation des échouages des algues en fonction des vents. Mais, presque tous les goémoniers, y compris les jeunes certifient que : 
«Tant que le goémon d'avril n'est pas arrivé à la côte, la dernière tempête de l'année n'est pas passée [...] Ici, rien n'est arrivé à la côte, du coup, c'est sûr qu'il allait y avoir un coup de vent. " (Un jeune goémonier vers Lanildut évoquant la météo peu clémente du mois d'avril 2014)

Entre la saison hivernale et la saison des algues, l'attention des goémoniers se porte sur l'avancement de la croissance des algues. Puisque l'observation de leur repousse est difficile et que l'algue est considérée comme une plante par la majorité des goémoniers, c'est sur la croissance des plantes terrestres que se basent les estimations. Avant les années 1960 , à l'époque des paysans-goémoniers ${ }^{15}$, l'attention se portait sur les cultures de blé ou de trèfle, aujourd'hui, ce sont les végétaux du quotidien dans «les haies", « les fougères sur les talus » qui servent d'étalon aux goémoniers :

«Tu n'as qu'à voir comment la végétation elle a poussé en 15 jours [...] Avec la luminosité, la température de l'eau automatiquement remonte aussi. On compare ça au trèfle, notre façon de voir les choses, c'est assez similaire aux paysans. Souvent, les anciens disaient quand le trèfle était bon, les laminaires étaient bonnes. Comme avant ils avaient une ferme, ils faisaient le goémon en plus, ils arrivaient à comparer facilement. Nous, c'est plus nos jardins, nos haies ou les champs pour savoir si les algues elles poussent parce que c'est juste une question de luminosité. » (Un des plus anciens goémoniers en activité)

Enfin, le début d'une «nouvelle petite saison» dans la saison des algues est identifié par les professionnels lorsque « le tosser commence à décrocher » fin août. « Le tosser » nom vernaculaire de Saccorhiza polychides (Figure 7) est la plus représentative selon les goémoniers des " algues indésirables » puisqu'ils la considèrent comme «l'ennemi des goémoniers et des laminaires ». Cette algue annuelle qui côtoie naturellement les ceintures de Laminaria digitata, biannuelle, participe à la dynamique des forêts d'algues brunes bretonnes. Pour les goémoniers, les «algues indésirables " correspondent à toutes les espèces récoltées involontairement et non valorisées par les entreprises de transformation. Il est indiqué dans les contrats qu'elles passent avec les goémoniers un pourcentage maximal de ces espèces à respecter dans le chargement, sous peine de pénalités financières. Le goémonier va donc s'appuyer sur un ensemble de savoirs naturalistes et techniques pour choisir des zones de travail «propres» et mettre en place une récolte respectant cet objectif. "Avoir une zone propre" est un des paramètres les plus importants avec l'accessibilité et les conditions climatiques qui dictent les déplacements, la fréquentation et la manière de travailler sur des lieux de récolte (Garineaud et al. 2016). Lorsque le tosser commence à dépérir, en raison de son cycle annuel, elle se détache des rochers laissant la place aux laminaires pluriannuelles. Ainsi, les goémoniers ont accès à de nouvelles zones de travail, mais cela annonce selon eux « les premiers coups de vent et de mer » empêchant la récolte des laminaires. Tous ces éléments sont centraux puisqu'ils conditionnent l'activité des goémoniers : où, quand, comment récolter les laminaires ? Les modifications environnementales causées par les changements climatiques viennent mettre à mal les repères des goémoniers ainsi que leur capacité à prévoir l'évolution de la ressource pour les saisons suivantes. 
Figure 7 : Saccorhiza polychides

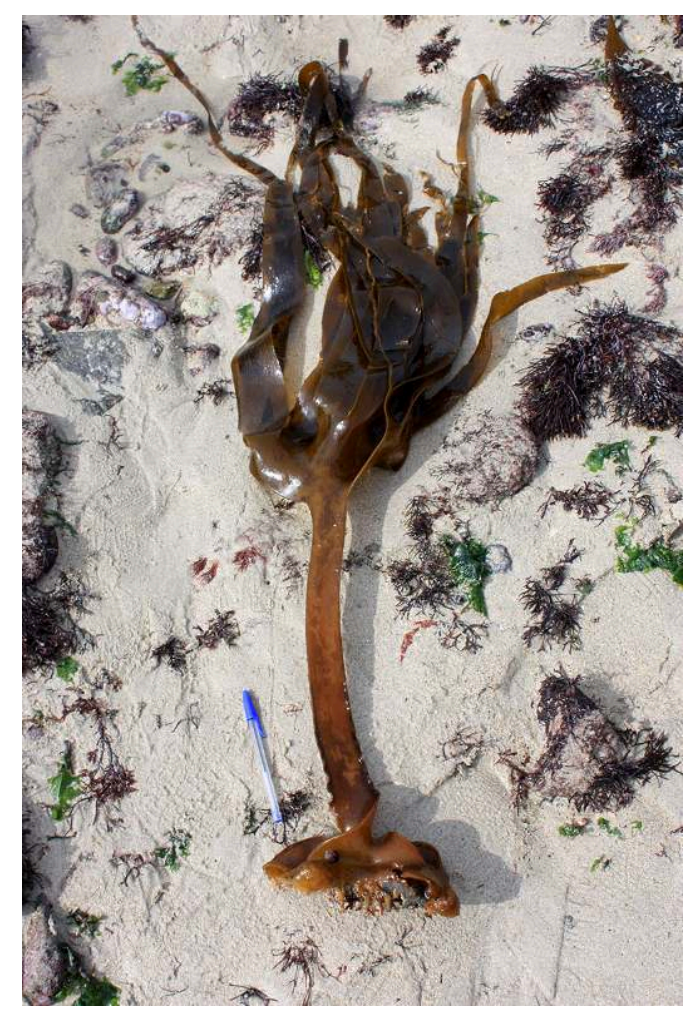

(c) C. Garineaud, 2014

\section{Prévoir au-delà de la saison}

Si les goémoniers sont capables en s'appuyant sur leurs observations et leurs savoirs d'anticiper l'évolution météorologique durant la saison de récolte, ils partagent également des réflexions très structurées reliant phénologie des algues, vents, météorologie et température de l'eau pour prévoir la saison suivante. Selon eux, pour faire démarrer la croissance des algues, la température de l'eau doit être froide. Ceci est possible lorsque le vent d'est apporte de la fraîcheur et des conditions météorologiques clémentes : la mer devient plus limpide, permettant aux algues d'accéder à la lumière nécessaire à leur croissance. Au contraire, le vent d'Ouest apporte des tempêtes où pluie et houle rendent l'eau turbide. Les propos de ce goémonier tenus en 2015 sur la saison 2016 à venir illustrent bien les liens établis entre ces paramètres :

«On est au début février, il y aura peut-être des tempêtes. Mais l'hiver n'a pas été violent. On a eu des coups de vent, mais il ne faut pas que ça dure. Il y a un manque de froid par contre. Il faut du froid pour l'agriculture, je crois que pour les algues c'est pareil. [...] Quand on a du froid, c'est des vents d'est et pas de pluie, mais du soleil. Qui dit lumière, dit photosynthèse donc ce qui en découle. C'est important pour les algues. Tout se lie. » (Un goémonier expérimenté)

En revanche, prévoir la qualité des récoltes à moyen voire à long terme est plus ardu en raison de la variabilité de la ressource. Pour certains goémoniers, la ressource en algue varie naturellement selon des "cycles répétitifs" sur deux temporalités : à moyen terme sur des durées de quatre à cinq ans et à long terme sur douze ou vingt-cinq ans. Les premiers cycles concernent le rétablissement des algues après une perturbation. Les seconds concernent uniquement la croissance des Laminaires et sont dits 
«naturels » par les goémoniers, car la ressource varie selon la dynamique naturelle de l'écosystème et des algues.

«Les vieux, ils m'ont dit: quand tu renverses quelque chose, il y en a pour 4 ou 5 ans à se refaire. Alors il va falloir attendre un peu et ça reviendra. Pourvu qu'il n'y ait pas d'autres hivers comme celui-ci. » (Un jeune goémonier)

Le découpage en cycles leur permet une certaine prévisibilité lorsqu'ils se souviennent d'années avec une faible biomasse. Les professionnels ne donnent pas d'explications sur les causes de ces variations, mais ils s'appuient des propos qui aurait été tenus par Pierre Arzel scientifique spécialiste des algues en Bretagne à l'IFREMER. Or, si ces perceptions sont relativement communes au sein des goémoniers, aucune publication ou travaux de Pierre Arzel ne fait état de ces cycles ${ }^{16}$. D'après les biologistes spécialisés dans les algues en Bretagne aujourd'hui actifs, aucune donnée scientifique dans les suivis biologiques ne confirme aujourd'hui l'existence de ces longs cycles.

Dans leur appréhension des changements, la mémoire permet de faire le lien entre ce qu'ils ont vécu, ce qu'ils observent dans la saison de récolte et ce qui s'annonce pour les saisons suivantes.

\section{Perception de changements locaux}

Alors que la variabilité de la ressource est un paramètre ancré dans les pratiques et les savoirs des goémoniers, il est intéressant de s'interroger sur le rapport entre celle-ci et les changements climatiques à l'échelle globale. Beaucoup de professionnels ont observé des changements à l'échelle locale, sur leurs territoires de pêche sans les associer aux changements globaux. Par exemple, les blooms de phytoplancton affectant leurs activités de pêche des coquillages ne sont pas reliés aux changements globaux. Nombre d'eux évoquent surtout le « réchauffement climatique » et ses conséquences comme ci-dessous. II peut s'agir d'évènements exceptionnels comme la présence de thon (Sarda sarda) dans les eaux douces de Bretagne.

"On trouve que la mer monte plus haut qu'avant et les saisons ne sont plus comme avant. Depuis quelques années, j'ai remarqué qu'on n'a plus les mêmes tempêtes ici, les vents commençaient sud et ça allait jusqu'à Norois ${ }^{17}$ et maintenant les vents, ils restent presque trois semaines Norois, chose qu'avant il n'y avait pas. Je ne sais pas, il y a sûrement quelque chose. Ça influence les algues et la pêche aussi. La température de l'eau, les gens ils disent que ça se réchauffe, je ne sais pas, mais il y a des choses qui se passent. L'année dernière, il y a eu du thon enfin c'est les bonites ${ }^{18}$ qui ont été pêchées. C'est le monde à l'envers. » (Un goémonier retraité)

Ces évènements affectent peu l'activité de récolte des algues. La perception des changements à l'échelle locale est variable selon les individus, une grande majorité de goémoniers bretons affirment ne pas savoir si les modifications qu'ils observent peuvent être associées à des changements globaux. Pour la majorité des goémoniers, la question du changement climatique ou du "réchauffement climatique " est un domaine réservé aux personnes qu'ils identifient comme "les scientifiques». Par ailleurs, jusqu'en 2014, l'abondance de la ressource, les conditions climatiques et la situation économique favorables ont réduit chez les goémoniers la dimension de variabilité qui caractérise les ressources aquatiques sauvages et la vulnérabilité qu'elle peut induire (Acheson 1981). L'aléa climatique de 2014 a mis fin à cette situation. 


\section{Esquisse de changements à venir et réponses adaptatives}

\section{Les tempêtes en 2014 révélatrices de la capacité d'adaptation des goémoniers}

«Les fonds sont blancs. On avait l'impression que les cailloux sont passés à la machine à laver, tout était blanc. On n'a jamais vu ça. Il n'y a plus de ressource. » (Un goémonier le jour de l'ouverture de la saison 2014)

L'hiver 2013-2014 a été particulièrement rude et exceptionnel. Une quinzaine de tempêtes se sont succédé en Bretagne, apportant des précipitations et des hauteurs de vagues records ${ }^{19}$. Cette situation météorologique a eu d'importantes conséquences humaines avec plusieurs décès dénombrés, mais aussi matérielles avec des inondations importantes faisant des dégâts majeurs ${ }^{20}$. Ces conditions climatiques ont diminué drastiquement la biomasse algale. Cette situation météorologique rare pourrait selon les scénarios climatiques proposés par le GIEC devenir de plus en plus fréquente (GIEC 2014). Elle fournit un aperçu d'une trajectoire future possible induisant une ressource présente en plus faible quantité et répartie de manière hétérogène. Cette situation inédite pour un grand nombre de goémoniers a permis d'observer les réponses adaptatives à l'échelle individuelle et du groupe des goémoniers. Les adaptations ont été de deux ordres : (1) une adaptation des techniques de récolte et des savoirs associés et (2) la production de nouveaux savoirs par l'apprentissage d'un autre métier et l'investissement de nouvelles zones de récolte. Ces changements rapides ont eu des répercussions tant sur le plan cognitif que sur le plan des régulations collectives de la récolte.

La première réponse des goémoniers à la faible croissance et à la répartition hétérogène des algues induite par les tempêtes a été une adaptation technique. Les goémoniers ont transformé leurs outils et techniques de travail : ils ont, par exemple, allongé la taille du scoubidou ou resserré sa base pour récolter plus profondément et atteindre des algues de petite taille. Ils ont légalement prolongé le temps de récolte d'environ trois heures par jour.

Ces changements techniques s'accompagnent de changements physiques et cognitifs. Dans un article précédent, nous avons démontré que les outils de récolte, le scoubidou et le peigne (Figure 6) sont des extensions corporelles qui communiquent avec l'individu comme tout organe, telle une prothèse sensorielle à travers les vibrations et les bruits (Garineaud 2015). Cette dimension sensorielle, fondamentale dans leur savoir-faire, va de pair avec des savoirs écologiques complexes. En effet, les goémoniers sont capables au travers des bruits, des vibrations et des mouvements du scoubidou et du bateau de savoir quels types et quelles quantités d'algues ils récoltent, et de caractériser les courants et les fonds marins, éléments indispensables à la pratique de leurs différents métiers (Garineaud 2015, Garineaud et al. 2016). La modification des outils en réponse aux changements de l'écosystème induit des fonctionnements différents : vibrations plus fortes, à-coups violents, etc. Additionnés à la fatigue en raison du temps de travail, plusieurs professionnels ont connu des problèmes musculaires. Ainsi, ces changements mécaniques, physiques et sensoriels, obligent le goémonier à réapprendre, ajuster et s'adapter pour conserver la correspondance entre ses savoirs sensibles, ses savoirs naturalistes et la réalité du milieu. 
34 telles qu'Himanthalia elongata ${ }^{21}$ (Figure 8) ou Laminaria hyperborea (Figure 4). Entre 2014 et 2015 , le nombre de bateaux récoltants cette dernière espèce a doublé. Dans un premier temps, les professionnels ont construit leur peigne (Figure 6) en se référant à ceux qu'ils avaient vus chez les autres goémoniers puis ils les ont rapidement modifié le poids, la taille et la forme ${ }^{22}$ - pour être plus adaptés à leurs bateaux et à leurs savoirs sensoriels. Ensuite, en s'appuyant sur leurs connaissances et en explorant, ils ont identifié les zones de récolte : il fallait savoir où aller, quand et comment récolter. En quelques semaines, ils ont appris souvent seuls, un métier requérant un savoir qui se construit au mètre près et jour après jour. Les savoirs expérientiels des goémoniers se sont révélés une ressource adaptative de premier ordre.

Figure 08 : Himanthalia elongata

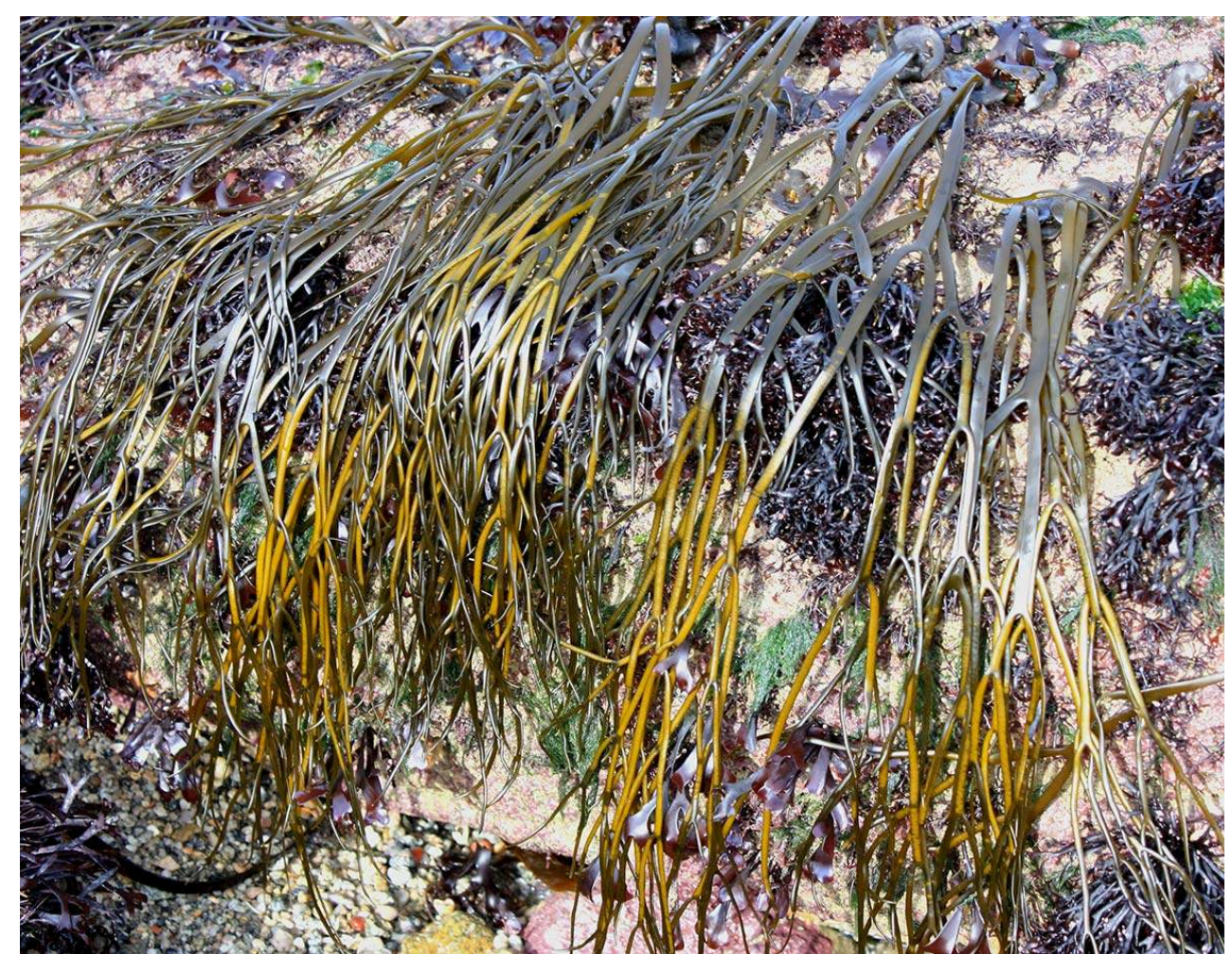

(c) C. Garineaud, 2014

déplacer vers de nouvelles zones alors que tous avaient, en période normale, assez de secteurs pour satisfaire leurs objectifs. Or, ce besoin d'être plus mobiles et plus flexibles s'est avéré doublement difficile pour les goémoniers habitués à leurs espaces historiques.

La première difficulté est liée à leurs savoirs, principale limite de leur efficacité de récolte (Geistdoerfer 1974). La modification de leurs savoirs (sur les espaces et les algues) et de leur savoir-faire a été très rapide, de l'ordre de quelques semaines et a affecté l'ensemble leurs activités, comme en témoigne le changement du système de classification des espaces de travail des goémoniers. En effet, les goémoniers utilisent un vocabulaire spécifique - « caillou, bordure, tache, roche et champs d'algues » - pour décrire à la fois les fonds marins, l'implantation des algues, organiser leurs aires de 
travail et décrire leurs pratiques de pêche que nous avons détaillée dans une publication précédente (Garineaud et al. 2016). Or, l'emploi de ce vocabulaire a été modifié temporairement en quelques semaines lors de la saison d'algues en 2014. Ainsi, le terme de " champ d'algue », représentant une longue et homogène étendue d'algue récoltée en se laissant dériver, a été remplacé par la " tache ", petite zone discontinue d'algues où il est nécessaire de s'arrêter en jetant l'ancre pour récolter. Ce changement de vocable s'est fait parallèlement au déclin de la biomasse, voire à la disparition complète des algues dans certains secteurs (Garineaud 2018). Ce changement sera probablement réversible avec le retour d'une situation normale. La seconde difficulté de cette mobilité forcée est qu'elle institue un nouveau fonctionnement collectif et de nouvelles règles de récolte. Les déplacements des secteurs d'activité ont provoqué des conflits entre les goémoniers du fait de la mise à mal d'accords tacites et anciens.

« Il y a quelques accords entre les goémoniers. Les goémoniers [de la commune 1], ils ont un coin là-bas, qui est pour les gens de [la commune1]. Ça date. Je n'étais pas né. Mais, ils essayent de venir sur [la commune2]. Il faut faire la police de temps en temps. Ça gueule, y a parfois des coups de fourches. Mais c'est comme ça. » (Un goémonier de la commune 2)

Des conflits sont également apparus entre goémoniers et pêcheurs au filet ou au casier qui partagent l'espace maritime.

Enfin, ces tempêtes ont eu pour conséquence de produire à l'échelle du groupe de professionnels une incertitude que nombre d'entre eux n'avaient jamais connue :

«Il y en a qui sont arrivés dans les algues pensant qu'ils allaient faire fortune tous

les jours. Et là, ils comprennent que non. » (Un goémonier retraité)

La mise en place de ces pratiques, parfois nouvelles, a obligé les goémoniers à sortir de leurs communautés de pratiques et à prendre en compte des savoirs construits à d'autres échelles par les différents acteurs de la filière. Ils ont notamment après des négociations entre eux et les usines de transformation - qui imposent le calendrier de récolte de Laminaria digitata décidé de retarder de 15 jours. Toutefois, le changement le plus important concerne leurs relations avec le monde " des scientifiques».

\section{Du changement dans les savoirs locaux et scientifiques}

Après les évènements de 2014, nous observons une différence au sein du groupe de professionnels sur la question de la perception des impacts des tempêtes localement et leurs liens envisagés avec les changements globaux. L'analyse des entretiens révèle que les goémoniers les plus anciens ou ceux ayant un membre de leur famille encore actif dans la profession sont moins disposés à évoquer ces changements globaux que les plus jeunes ou ceux n'ayant pas de famille pratiquant la récolte des algues. Si, nous constatons une légère évolution chez les plus anciens qui évoquent d'autres saisons difficiles, ils restent dans un raisonnement par cycle. En revanche, les jeunes goémoniers qui n'ont plus de membre de leurs familles en activité apparaissent plus aux familiers et demandeurs d'informations sur les changements globaux que les autres goémoniers s'inscrivant dans une tradition familiale.

41 La construction des savoirs et leur type de transmission ou d'acquisition semblent essentiels dans la prise en compte de la notion de changements climatiques globaux. Mariana Bender et ses collègues soulignent dans leurs travaux sur des communautés de pêcheurs du Brésil que des ruptures générationnelles accroissent la vulnérabilité en raison des difficultés des pêcheurs à reconnaître les tendances environnementales à 
long terme (Bender et al. 2013). La différence que nous notons pourrait être liée aux parcours personnels plus diversifiés - études plus longues, expériences professionnelles hors de la pêche - pour les jeunes goémoniers ou ceux n'ayant pas de famille pratiquant la récolte des algues. Pour les professionnels plus âgés sans membre de leur famille dans la profession, la période de transmission est très courte puisqu'elle dure en moyenne 5 jours. Celle-ci se focalise sur les techniques, la connaissance des zones de récolte, mais peu sur les savoirs liés à la ressource en elle-même. C'est donc seul que le goémonier fait l'apprentissage du métier créant des ruptures dans la transmission des savoirs.

Suite aux tempêtes de 2014, une évolution dans les interactions avec les biologistes et écologues peut être observée. Tous les goémoniers sont amenés à échanger 3 ou 4 fois par an lors des réunions de bilan et de préparation de la saison de récolte. La confrontation de communautés épistémiques, goémoniers face aux scientifiques, s'est transformée en un échange entre ces deux groupes favorisant l'émergence d'un savoir pouvant être qualifié d'hybride (Dove 2002 ; Dumez 2010). Cette hybridité se construit par un processus intellectuel d'intégration des savoirs d'autres acteurs de manière délibérée ou involontaire. Différents degrés d'intégration existent que nous retrouvons chez les goémoniers: soit la personne identifie les savoirs comme différents du sien, mais les mobilise, soit elle combine plusieurs types de savoirs d'où émerge un nouvel ensemble épistémologique. Le premier type - savoir composite - est fréquent, mais les problématiques liées au changement climatique sont un terrain fertile pour l'émergence d'un second type de savoir, un savoir hybride. Les propos d'un jeune goémonier illustrent le premier type de savoir :

«Les laminaires, c'est des algues qui vivent dans des eaux froides donc s'il y avait une augmentation de la température, il y a modification de l'environnement. C'est ce qu'avait remarqué Pierre Arzel dans ses livres. D'après lui, dans le temps, on verrait une diminution dans le sud de la Bretagne de digitata. Et là, moi je vois aussi que la mer a du mal à descendre en température. Là, c'est autour de douze, il faudrait que ça perde un ou deux degrés de plus. [Aujourd'hui, le scientifique de l'Ifremer] en parle un peu dans les réunions. Je ne suis pas habilité. J'en parle parce qu'il m'en a parlé [récemment], ce n'est pas moi qui l'ai inventé.» (Un jeune goémonier en 2016)

Les goémoniers ont repris des éléments du langage des scientifiques impliqués dans le suivi de la récolte des algues. La similarité de leurs discours est flagrante comme le montre l'exemple sur la biologie de Laminaria hyperborea et seul l'usage de termes issus de la botanique et de la phycologie (latence, canopée, etc.) permet réellement de faire

\begin{tabular}{c|c} 
la $\begin{array}{c}\text { différence } \\
\text { Chercheur de l'lfremer }\end{array}$ & les deux \\
Goémonier embarqué
\end{tabular}

Pour ces scientifiques, de l'IFREMER ou de laboratoires universitaires bretons, l'échange n'est pas unidirectionnel, contrairement à ce qu'avancent les goémoniers, puisque les pêcheurs, par leurs observations sur le terrain, apportent, « la connaissance locale, la connaissance territoriale" (un biologiste de l'IFREMER impliqué dans la gestion des algues). Ces propos concernaient l'effet des tempêtes de 2014 et témoignent de l'importance des échanges informels dans les ports ou lors des réunions annuelles avec les goémoniers. Le chercheur explique que les informations recueillies au fur et à mesure sur les zones impactées par la tempête lui ont permis d'analyser les différents 
facteurs aggravants la perte de la biomasse et d'en tirer des conclusions qui viennent conforter ses observations et hypothèses :

«Les goémoniers m'ont dit qu'à partir de telle profondeur il n'y a pas de digitata. Pourquoi ? Manque de lumière, problème de croissance. Par contre là où ils pêchent bien, c'est sur les parties hautes ou sur la roche mère. Là où il $\mathrm{y}$ a des manques de biomasse, c'est là où il n'y a pas de roche mère, dans les situations de blocs. Une partie de l'habitat s'est retrouvé avec une force cinétique et il y a un phénomène de retournement de blocs et donc une érosion des jeunes plants. En début de saison, je ne pouvais pas avoir cette analyse-là, car je n'avais pas cette information-là puisque les goémoniers n'avaient pas été suffisamment sur le terrain pour avoir un regard très précis. C'est là où le savoir est partagé. » (Un scientifique de l'ffremer)

Ce partage apparaît alors indispensable pour les scientifiques pour analyser les situations délicates ou faire émerger des problèmes et compense les difficultés à couvrir un large territoire par des relevés de terrain, car il est difficile pour les scientifiques d'analyser par manque de temps et de moyens l'ensemble des milieux où les goémoniers travaillent. Il permet également de favoriser le dialogue donc d'établir les modalités de gestion les plus adaptées notamment aux changements futurs :

«On partage la connaissance et le savoir à partir duquel on fait des choix et des propositions d'orientation [...] Je trouve ça bien qu'il y ait des positions contradictoires, ce n'est pas que ça remet en cause, mais ça indique aux représentants comme moi, il faut que mes résultats soient plus probants, améliorer mon travail pour qu'il y ait moins d'incertitude dans la connaissance. Là où il y a moins d'incertitude, les résultats sont plus partagés par les acteurs. On peut alors décider de façon plus collégiale de mesures et même ça peut venir d'eux. » (Un scientifique qui collabore avec les goémoniers embarqués interrogés sur l'échange de connaissances)

L'hybridation entre les savoirs locaux et les savoirs scientifiques présente partiellement avant, a été fortement accentuée par l'aléa de 2014 et semble favoriser l'adaptation aux changements climatiques. Toutefois, durant la saison 2014, les acteurs industriels ont fait preuve de réticences à modifier les modalités de la récolte - dates d'ouvertures, tonnage journalier et pourcentage d'algues non désirées - malgré le consensus sur ces points entre goémoniers et scientifiques. Les conditions climatiques favorables les années suivantes ont permis un retour de la ressource et une situation plus confortable. Mais, face à une situation aujourd'hui exceptionnelle qui pourrait devenir plus fréquente, une interrogation majeure émerge sur l'adéquation entre les demandes des industriels, les choix politiques et les dynamiques naturelles. Interrogation à laquelle une anthropologie des changements climatiques plus longue et globale pourrait répondre.

\section{Partir du local pour saisir le changement global}

La question des changements est une réalité émergente sur le terrain. Il est nécessaire de l'intégrer directement dans les questionnements, mais cela demande une connaissance fine, au préalable, des groupes sociaux et de l'environnement étudié. En ce sens, l'analyse des pratiques et des savoirs locaux est une approche pertinente. Elle permet de saisir les lacunes, comme sur les coquillages et les blooms de phytoplancton, la perception des changements à l'œuvre localement et les mécanismes d'adaptations mis en place par les groupes étudiés, au travers de la parole et des observations du quotidien ou lors d'évènements climatiques exceptionnels. 
47 Notre analyse montre que le riche cortège de savoirs locaux qui permet aux goémoniers de prévoir l'évolution de la ressource et intègre la variabilité naturelle. La prise en compte des changements à plus grandes échelles ne faisait pas partie d'une réalité tangible pour une partie des goémoniers. L'aléa climatique de 2014 est apparu comme un révélateur de savoirs et des dynamiques locales. Il a obligé les professionnels à s'adapter, montrant leurs capacités d'adaptation à court terme. Les savoirs locaux s'inscrivaient avant 2014 dans un dialogue ponctuel avec des savoirs scientifiques. Face à la crise de 2014, ce dialogue s'est intensifié, conduisant à l'élaboration d'un savoir que nous qualifions d'hybride apparaissant alors comme une ressource pour les goémoniers afin d'appréhender la réalité des changements globaux et renforcer leur capacité d'adaptation.

Au-delà des éléments ethnographiques, notre réflexion souhaite contribuer aux questionnements de l'ethnoécologie (Dumez et al. 2014), de l'anthropologie maritime (Reyes et al. 2018) et de l'anthropologie des changements climatiques (Sourdril \& Garine 2019) qui tente de saisir les interactions entre les sociétés humaines et un environnement - marin - en perpétuels changements.

Les changements climatiques, l'anthropocène ne sont pas les termes des communautés locales. Ils émanent des discours scientifiques et sont relayés par les gouvernements, les médias ou autres. Il faut donc avoir une double analyse. La première est la définition, la réalité et l'appropriation de ces concepts par les acteurs extérieurs aux communautés étudiées. La seconde est l'ethnoclimatologie indissociable de l'ethnoécologie.

50 Toutefois, l'appréhension des questions climatiques posent deux questions celle de la durée et de l'échelle (Sourdril \& Garine 2019). Ornella Puschiasis (2019) dans son article met bien en évidence la nécessité d'un temps long afin de révéler les indicateurs calendriers traditionnels, le rapport aux évènements extrêmes et les savoirs rarement verbalisés directement. En accord avec cette conclusion, notre enquête s'est prolongée, pour saisir l'évolution des situations et il a été nécessaire d'intégrer des échelles plus grandes. Enfin, l'anthropologie des changements climatiques ne peut échapper à la question de l'adaptation demandant une nouvelle fois un temps long de présence et une analyse large des facteurs externes. Toutes ces réflexions se retrouvent dans notre cas d'étude puisque suite aux évènements extrêmes de 2014, l'instance de gestion de la récolte des algues a commencé à se questionner et à se réorganiser sur son fonctionnement. Notre analyse des systèmes de savoirs limitée dans le temps n'a pas permis d'appréhender ces transformations qui portent sur la mise en place de nouveaux rapports dans les instances de décisions et de gouvernance: situation de solidarité, jeux de pouvoir, remise en cause de la réglementation officielle et tacite, sur le renouvellement ou non-modalités de gestions. Si l'activité goémonière a été en mesure de s'adapter - et cela est toujours en cours - après l'aléa de 2014, au regard de la communauté restreinte des acteurs, de la construction de la filière (écoulement et débouchés), des changements rapides dans les écosystèmes, cette adaptation ne présage pas de sa capacité à s'adapter face à une épreuve future écologique ou économique plus intense et plus globale. La dimension économique prend dans ce contexte une ampleur différente. Comme le souligne Tarik Dahou, l'appropriation immatérielle et matérielle de la nature est désormais influencée par des imbrications complexes et des dynamiques globales notamment du fait de la soumission des usages de la nature aux effets directs ou indirects du libéralisme (Dahou 2018). Dans la 
situation étudiée, les deux multinationales acheteuses d'algues ont accepté de réduire leurs demandes exceptionnellement. Mais, il faut s'interroger si cette situation venait à se reproduire ou à se pérenniser, sur la position qu'elles adopteraient. Cela laisse entrevoir la mainmise de l'économie globale sur le système de gestion local.

51 Ainsi, l'enquête anthropologique sur les changements climatiques ne doit pas se limiter aux spatialités et aux temporalités du groupe social étudié, mais elle doit être multisituée, prendre en compte la temporalité institutionnelle, les enjeux économiques tout en amenant d'autres manières d'enquêter, corpus et concepts.

\section{BIBLIOGRAPHIE}

Acheson J. 1981 - Anthropology of Fishing. Annual Review of Anthropology 10 (1) : 275-316.

Adger W.N., Hughes T., Folke C., Carpenter S. \& Rockström J. 2005 - Social-Ecological Resilience to Coastal Disasters. Science 309 (5737) : 1036-1039.

Artaud H. 2011 - La poiétique des flots. Thèse. Écoles des Hautes Études en Sciences Sociales, 352 p.

Artaud H. 2018 - Anthropologie maritime ou anthropologie de la mer ? Revue d'ethnoécologie 13 [En ligne] http://journals.openedition.org/ethnoecologie/3484_Consulté le 15 mars 2021.

Arzel P. 1987 - Les goémoniers. Douarnenez, Chasse-marée, 305 p.

Baer H. \& Reuter T. 2015 - Anthropological Perspectives on Climate Change and Sustainability: Implications for Policy and Action. Brief for Global Sustainable Development Report : 1-3.

Bender M. Floeter S. \& Hanazaki N. 2013 - Do traditional fishers recognise reef fish species declines? Shifting environmental baselines in eastern Brazil. Fisheries Management and Ecology 20 (1) : 58-67.

Biget D. 2005 - Des écoles de pêche aux lycées maritimes et aquacoles. Techniques \& Culture 45 (Juin) : 1-14.

Collectif 2017 - La filière des algues en France. Evolution et poids économique. Synthèse Agrocampus Ouest, [En ligne] https://idealg.u-bretagneloire.fr/sites/default/files/documents/ pole_halieutique_2018.pdf. Consultée le 05 aout 2019.

Crate S. \& Nuttall M. 2009 - Introduction : Anthropology and Climate Change. In Crate S. \& Nuttall M. (Ed.) Anthropolgy and Climate Change, From Encounters to Actions. Walnut Creek - CA, Left Coast Press : 9-35.

Dahou T. 2018 - Gouverner la mer en Algérie, politique en eaux troubles. Paris, Kartala, 248 p.

Delbos G. 2006 - Pêche artisanale : la fin du « ménage ». Ethnologie Française 36 (3) : 531-42.

Delegrange A., Lefebvre A., Gohin F., Courcot L. \& Vincent D. 2018 - Pseudo-nitzschia sp. diversity and seasonality in the southern North Sea, domoic acid levels and associated phytoplankton communities. Estuarine Coastal and Shelf Science 214 : 194-206. 
Dond S. \& Manyungwa-Pasani C. 2018 - Understanding indigenous knowledge: its role and potential in fisheries resources management in Malawi. Aquatic Ecosystem Health \& Management 21 (2) : 176-84.

Dove M. 2002 - Histoires et savoirs autochtones hybrides chez les petits cultivateurs d'hévéa d'Asie. Revue Internationale Des Sciences Sociales 173 (3) : 389-400.

Dove M. 2014 - The Anthropology of Climate Change: An Historical Reader. Chichester, John Wiley \& Sons, $360 \mathrm{p}$.

Doyon S. \& Sabinot C. 2015 - Anthropologie des espaces côtiers et de la conservation environnementale. Pêche, sel et flamands roses dans les Réserves de biosphère yucathèques au Mexique. Laval, Presses de l'Université Laval, $322 \mathrm{p}$.

Dumez R. 2010 - Le feu, savoirs et pratiques en Cévennes. Versailles, Quae, 248 p.

Dumez R., Roue M. \& Bahuchet S. 2014 - Conservation de la nature : quel rôle pour les sciences sociales? Revue d'ethnoécologie 6 [En ligne] URL : http://journals.openedition.org/ethnoecologie/ 2089. Consulté le 15 mars 2021.

De Freitas J.G., Bastos R. \& Alveirinho Dias J. 2018 - Traditional Ecological Knowledge as a Contribution to Climate Change Mitigation and Adaptation: The Case of the Portuguese Coastal Populations. In Leal Filho W., Manolas E., Azul A.M., Azeiteiro U.M. \& McGhie H. (Ed.) Handbook of Climate Change Communication: Vol. 3: Case Studies in Climate Change Communication. SpringerBook : 257-269.

Folke C., Carpenter S., Elmqvist T., Gunderson L., Holling S. \& Walker B. 2002 - Resilience and sustainable development: building adaptive capacity in a world of transformation, AMBIO A Journal of the Human Environment 31 (5) : 437-40.

Frangoudes K. \& Garineaud C. 2015 - Governability of kelp forest small-scale harvesting in Iroise sea, France. In Jentoft S. \& Chuenpagdee R. (Ed.) Interactive governance for small-scale fisheries. Amsterdam, MARE Publication Series : 101-115.

Gallon R., Robuchon M., Leroy B., Le Gall L., Valero M. \& Feunteun E. 2014 - Twenty years of observed and predicted changes in subtidal red seaweed assemblages along a biogeographical transition zone: inferring potential causes from environmental data. Journal of Biogeography 41 (12) : 2293-2306

Garineaud C. 2015 - Pratiques manuelles ou mécanisées : la part de la main dans les perceptions sensorielles et dans les savoirs écologiques. Exemple des récoltants d'algues en Bretagne. Ethnographie.org 31 [En ligne] URL : http://www.ethnographiques.org/2015/Garineaud. Consulté le 4 juin 2020.

Garineaud C. 2017 - Récolter la mer : des savoirs et des pratiques des collecteurs d'algues à la gestion durable des ressources côtières dans le Finistère (Bretagne). Thèse, Muséum National d'Histoire Naturelle de Paris, 330 p.

Garineaud C. 2018 - Evolving in changing environment: practices and knowledges adaptability of seaweed harvesters in Brittany. AMBIO A Journal of the Human Environment : 1-20.

Garineaud C., Levain A., Feunteun E. \& Dumez R. 2016 - Le caillou, le champ et la forêt représentations et appropriations des espaces par les goémoniers dans le Finistère. In Robert S. \& Melin H. (Ed.) Habiter le littoral, enjeux contemporains. Presse Universitaire Aix-en-Provence : 125-139.

Geistdoerfer A. 1974 - Savoir et techniques des pêcheurs des îles de la Madeleine (Québec). Journal d'Agriculture Tropicale et de Botanique Appliquée 21 (7-9) : 169-217. 
Geistdoerfer A. 2004 - De l'origine des marins. La genèse mythique d'une spécialisation technique. Techniques et Culture 43-44:2-13.

GIEC 2014 - Climate change 2014: impacts, adaptation, and vulnerability. Part A: global and sectoral aspects. Contribution of working group ii to the fifth assessment report of the intergovernmental panel on climate change. Report 2014, Geneva Switzerland, 1120 p.

Godard O. 2010 - Cette ambiguë adaptation au changement climatique. Natures Sciences Sociétés, (18) : 287-97.

IFREMER 2018 - L'approche par pêcherie : définition de l'Ifremer. Rapport ressources halieutiques, exploitation durable et valorisation : 1-4.

Jacquin P. 1980 - Le goémonier. Paris, Berger-Levrault, 115 p.

Jacquin P. 2000 - La guerre des algues. Contestations et affrontements pour le partage de l'estran dans la France de l'Ouest. In Le Bouëdec G. \& Chappe F., Pouvoirs et littoraux du XVe au XXe siècle. Presses universitaires de Rennes : 617-622.

Johannes R. 1981 - Working with fisherman to improve tropical coastal fisheries and resource management. Bulletin of Marine Science 31 (3) : 673-80.

Jorion P. 1979 - Les deux concepts fondamentaux de la pêche artisanale : la « saison » et le « métier ». Ethnologie Française 9 (2) : 135-146.

Jorion P. 1983 - Les Pêcheurs d'Houat : anthropologie économique. Paris, Hermann, 198 p.

Keith S., Kerswell A. \& Connolly S. 2014 - Global diversity of marine macroalgae: environmental conditions explain less variation in the tropic. Global Ecology and Biogeography 23 (5) : 517-29.

Nakashima D. \& Roué M. 2002 - Indigenous knowledge, peoples and sustainable practice. Encyclopedia of Global Environmental Change (5) : 314-24.

Narchi N.E., Cornier S., Canu, D.M., Aguilar-Rosas L.E., Bender M.G., Jacquelin C. \& de Wit R. 2014 - Marine ethnobiology a rather neglected area, which can provide an important contribution to ocean and coastal management. Ocean and Coastal Management 89 : 117-126.

Ojea E., Pearlman I., Gaines S. \& Lester S. 2017 - Fisheries Regulatory Regimes and Resilience to Climate Change. Ambio A Journal of the Human Environment (46): 339-412.

Pecl G., Araujo M., Bell J., Blanchard J. \& Bonebrake T. 2017 - Biodiversity redistribution under climate change: Impacts on ecosystems and human well-being. Science 355 (6332) : 1-9.

Puschiasis O. 2019 - Un vent de changements souffle sur l'Everest. Multiples facettes de la perception de la météorologie et du climat chez les Sherpa. Ethnographiques.org, 38 [En ligne] URL :https://www.ethnographiques.org/2019/Puschiasis. Consulté le 15 mars 2021.

Raybaud V., Beaugrand G., Goberville E., Delebecq G., Destombe C. \& Valero C. 2013 - Decline in kelp in west Europe and climate. PLoS ONE 8 (6) : 1-10.

Reyes N., Garineaud C. \& Chlous F. 2018 - Défis scientifiques et sociaux de l'anthropologie maritime. Revue d'ethnoécologie 13 [En ligne] URL : http://journals.openedition.org/ ethnoecologie/3485. Consulté le 11 mars 2021.

Roué M. 2012 - Histoire et épistémologie des savoirs locaux et autochtones. De la tradition à la mode. Revue D'ethnoécologie (1) : 1-14.

Simonet G. 2009 - Le concept d'adaptation : polysémie interdisciplinaire et implication pour les changements climatiques. Natures Sciences Sociétés (17) : 392-401. 
Sourdril A. \& Garine E. 2019 - Comment parler du temps qu'il fait à l'heure du changement climatique ? Petit catalogue de « Météorologies locales ». Ethnographiques.org 38 [En ligne] URL https://www.ethnographiques.org/2019/Sourdril_Garine._Consulté le 14 mars 2021

Valero M., Destombe C., Mauger S. \& Ribout C. 2011 - Using genetic tools for sustainable management of kelps : a literature review and the example of Laminaria Digitata. Cahiers de Biologie Marine (52) : 467-83.

\section{NOTES}

1. D’après Bates, cité par Simonet 2009 : - « processus par lequel les organismes ou populations d'organismes effectuent des ajustements biologiques ou comportementaux qui facilitent ou assurent leur succès reproducteur, et donc leur survie, dans leur environnement ».

2. Le quatrième rapport du Groupe d'experts intergouvernemental sur l'évolution du climat (GIEC) a indiqué que la température globale à la surface de la mer (SST) a augmenté en moyenne de $0,13^{\circ} \mathrm{C}$ par décennie au cours des 50 dernières années.

3. Nous entendons par savoir locaux : «issus d'une démarche holistique, les savoirs locaux sont un corps cumulatif de savoirs et un ensemble d'observations empiriques sur l'environnement local - pouvant inclure des systèmes de classification -, lié à des pratiques (savoir-faire), à une éthique et à des représentations du monde (visible et invisible), portant sur les relations entre les humains, les non humains et leur monde. Ces savoirs locaux sont transmis de génération en génération comme un héritage culturel et ils évoluent par l'incorporation dans le tissu social et dans les pratiques de nouvelles techniques et d'innovations. » (Garineaud $2017: 17$ )

4. Cette recherche a bénéficié du soutien financier de la Fondation de France, dans le cadre du programme Environnement « Quels littoraux pour demain ? » entre 2014 et 2017

5. Une dizaine de terrains d'une durée variant de deux semaines à huit mois sur place ont été réalisés durant toutes saisons pour accompagner le plus longtemps possible les professionnels dans leurs activités.

6. L'Institut Français de Recherche pour l'Exploitation de la Mer est un établissement public à caractère industriel et commercial qui a pour objectif de produire de la connaissance et d'évaluer les ressources des océans et permettre leur exploitation durable.

7. Le comité régional des pêches maritimes et des élevages marins un organisme qui représente et défend les intérêts des marins.

8. Selon l'expression en usage chez les goémoniers. Ils évoquent sous ce nom les lycées professionnels maritimes qu'ils ont fréquentés.

9. Jusqu'aux années 1950-1960, la récolte se faisait à la main avec des bateaux pour les hommes et à pied pour les femmes. Cette activité indissociable de la ferme a construit l'identité du paysangoémonier décrit entre autres par Pierre Arzel. La mécanisation des techniques et le bouleversement $\mathrm{du}$ monde agricole ont transformé profondément la récolte des algues conduisant au fonctionnement actuel.

10. Une seule a pu être rencontrée. Si elle ne pratique plus cette activité aujourd'hui pour des raisons personnelles, elle s'inscrit dans un parcours proche de celui des jeunes goémoniers et elle partage les savoirs des autres goémoniers.

11. La recherche a été menée dans un réseau restreint d'acteurs $(\mathrm{N}=50)$ et très localisée. Afin que les professionnels puissent parler librement, il nous revient de garantir la confidentialité de leurs propos et leur anonymat. Nous avons fait le choix d'indiquer au lecteur s'il s'agissait d'un jeune goémonier nouvellement arrivé dans la profession ou d'un professionnel plus expérimenté, voire retraité. 
12. Il existe très peu d'informations économiques accessibles sur la filière d'alginate. Les deux entreprises sont Dupond de Nemours, implantée depuis 10 ans, à Landerneau et Algaia qui a racheté l'usine de Cargill en 2016 de Landéda.

13. Il s'agit de l'unité de mesure de la vitesse d'une embarcation. Il correspond à un mille nautique par heure, soit 1,852 kilomètre par heure.

14. Cf. note $\mathrm{n}^{\circ} 9$

15. Cf. note $n^{\circ} 9$

16. Pierre Arzel étant décédé en 2008, je n'ai pas eu l'occasion de lui poser la question. J'ai en revanche, grâce à sa compagne, pu accéder à ses notes et archives personnelles.

17. Le Norois est un vent de secteur nord-ouest.

18. Bonite à dos rayé, espèce : Sarda sarda

19. D'après Météo-France, les bilans climatiques de l'hiver 2013-2014 montrent que la pluviométrie été deux fois supérieures à la normale. Lors des tempêtes, les hauteurs moyennes et maximales de la houle et des marées ont été aussi exceptionnelles. C'est au mois de février que les hauteurs significatives des vagues mesurées au large du Finistère ont atteint leurs apogées avec environ douze mètres et jusqu'à dix-neuf mètres.

20. 14 février 2014, le magazine Le Point publiait un article illustrant parfaitement la situation dans laquelle notre terrain de thèse débuta. Il commençait comme cela : « Tempêtes : les Bretons n'en peuvent plus. Dernière en date, la tempête Ulla a fait un mort et laisse encore des dizaines de milliers de foyers sans électricité. Et ça fait deux mois que ça dure !»

21. Cette espèce est ramassée par un autre groupe de professionnels qui récoltent des algues à pied avec des faucilles ou à la main. Cette algue s'insère dans une autre filière économique (transformation alimentaire ou pharmacologique) jusqu'alors délaissée par les goémoniers en raison des faibles volumes non rentables.

22. Ils ont notamment ajusté le nombre de doigts (cf. Figure 6) ou l'écartement entre ceux-ci

\section{RÉSUMÉS}

Les côtes bretonnes abritent une diversité algale exceptionnelle affectée par les changements climatiques mettant à mal des activités de récolte traditionnelles. Les goémoniers sont directement concernés et sont des observateurs privilégiés de ces changements. À partir de matériaux ethnographiques et ethnoécologiques, cet article interroge la manière dont se formulent localement les savoirs locaux, la perception des changements et leur articulation avec les changements globaux. Les goémoniers possèdent un riche et complexe cortège de savoirs locaux adaptés pour prévoir et récolter la ressource en tenant compte de sa variabilité. Si l'appréhension des changements globaux était faible chez les goémoniers, ces savoirs apparaissent comme des indicateurs des changements locaux. L'aléa climatique exceptionnel en 2014, esquisse potentielle du changement à venir, a été révélateur de la capacité d'adaptation des professionnels à court terme en produisant de nouveaux savoir-faire et savoirs. Une des transformations est l'intensification des interactions entre les acteurs scientifiques et les goémoniers avec la construction d'un savoir hybride. Bien que l'approche par les savoirs fournisse des éléments précis sur la réalité du changement climatique chez les goémoniers, elle se trouve contrainte pour saisir toutes les transformations profondes notamment dans les instances de gouvernance et permettre ainsi la réalisation d'une anthropologie du changement climatique holiste. 
Brittany's coastline has an exceptional diversity of algae, which is nowadays impacted by climate change, thus affecting the harvesting activities that have been carried out for several centuries. The seaweeds harvesters who collect the algae are directly concerned and they are also privileged observers. Based on ethnographic and ethnoecological material, this article examines the local knowledge, the perception of changes and their articulation with climates changes. The seaweeds harvesters have developed a rich and complex local knowledge that hels them predict and harvest the resource, taking into account its variability. Though the apprehension of climate change was low among harvesters, this knowledge appears as an indicator of local changes. The exceptional climatic hazard in 2014, a potential outline of the upcoming change, has revealed the adaptive capacity, in the short term, of professionals, producing new know-how and knowledge. One of the transformations is the intensification of interactions between scientific actors and harvesters with the development of hybrid knowledge. Although the knowledge-based approach provides precise information on the reality of climate change among seaweeds harvesters, it is constrained to understand all transformations, particularly in governance, and to enable the realization of a holistic anthropology of climate change.

\section{INDEX}

Keywords : seaweeds harvester, Brittany, Traditional Ecological Knowledge, climate change, adaptation

Mots-clés : goémonier, Bretagne, savoirs locaux, changement climatique, adaptation

\section{AUTEUR}

\section{CLÉMENT GARINEAUD}

Chercheur associé UMR 7206 Ecoanthropologie, Muséum national d'Histoire naturelle 